where $A(0)=0, B(1)=0$,

$$
\begin{aligned}
& A(x)^{(K-1) / K}+B(x)^{(K-1) / K}=C, \\
& A^{\prime}(x) B(x)-B^{\prime}(x) A(x)=1, \\
& \ln C=-\frac{K-1}{2 K} \ln \left(\frac{K}{K-1} \frac{[\Gamma(K /(K-1))]^{2}}{\Gamma(2 K /(K-1))}\right) .
\end{aligned}
$$

(8) Substituting (13) and (14) back into the expression for $W[F]$ and integrating by parts gives

$$
W\left[F_{0}\right]=-1-2 K \ln C \text {, }
$$

and substituting this result into (10) recovers the formula in (4) for the behavior of $A_{n}$ when $n$ is large.

We are currently working on extending these methods to higher-dimensional field theories and hope to use them eventually to obtain the radius of convergence of $F_{1}(\alpha)$.

*Alfred P. Sloan Research Fellow。Work supported in part by National Science Foundation under Grant No. 29463. †Work supported in part by the U. S. Energy Research and Development Administration under Contract No. AT(111) -3227 .

${ }^{1}$ S. L. Adler, Phys. Rev. D $\underline{5}, 3021$ (1972). This paper contains references to the earlier work of M. Gell-Mann, F. E. Low, K. A. Johnson, M. Baker, R. Willey, and others who have examined the problem of finite electrodynamics.

${ }^{2}$ See J. Rosner, Phys. Rev. Lett. 17, 1190 (1966), and Ann. Phys. (N.Y.) $\underline{44}, 11$ (1967), for the calculation of the coefficient of $\alpha^{3}$.

${ }^{3}$ C. M. Bender and T. T. Wu, Phys. Rev。Lett. 27, 461 (1971)。

${ }^{4} \mathrm{C}$. M. Bender and T。T.Wu, Phys。Rev。D $\underline{7}, 1620$ (1973).

${ }^{5}$ Similar investigations were also carried out by T. Banks, C. M. Bender, and T. T. Wu, Phys. Rev. D 8 , 3346 (1973); T. Banks and C. M. Bender, Phys。Rev. D $\underline{8}, 3366$ (1973), and J. Math. Phys. (N.Y.) 13, 1220 (1972).

${ }^{6}$ Plots of these distributions are given for various values $n$ by $C_{0} M_{0}$ Bender and T。T。Wu, to be published. We thank $\mathrm{Mr}$. Robert Keener for his assistance in preparing these plots.

${ }^{7} \mathrm{~A}$ careful treatment of propagators which are not simple exponentials is given in Ref. 6 .

${ }^{8}$ The use of Laplace's method here emphasizes that the behavior of perturbation theory in large order reflects the semiclassical content of the theory。 A brief discussion of this point is given in Ref。1.

\title{
Phase Transitions in the Quantum Heisenberg Model
}

\author{
Freeman J. Dyson* \\ Institute for Advanced Study, Princeton, New Jersey 08540
}

and

Elliott H. Lieb† and Barry Simon

Departments of Mathematics and Physics, Princeton University, Princeton, New Jersey 08540

(Received 10 May 1976)

\begin{abstract}
We rigorously prove that in three or more dimensions, the nearest-neighbor, simplecubic, ferromagnetic, quantum Heisenberg model of spin $S\left(=\frac{1}{2}, 1, \cdots\right)$ has a phase transition at nonzero temperature.
\end{abstract}

The quantum Heisenberg model represents one of the simplest models in which ferromagnetic behavior occurs. From a variety of intuitions including high-temperature expansions ${ }^{1}$ and spinwave approximations, ${ }^{2}$ it has been believed for many years that the three-dimensional model has a first-order phase transition in the magnetic field at sufficiently low temperatures. Despite its obvious physical interest, a rigorous proof of this has not been available-the only previous rigorous results on phase transitions in quantum lattice systems are proofs of the absence of phase 
transitions in suitable models in external field, ${ }^{3}$ the proof of absence of long-range order in twodimensional isotropic models, ${ }^{4}$ and the proof of a phase transition for highly anisotropic models. ${ }^{5}$ In this Letter we wish to announce and sketch a rigorous proof of the occurrence of spontaneous magnetization at nonzero temperatures in nearest-neighbor, simple-cubic, ferromagnetic quantum Heisenberg models in three or more dimensions.

To describe the $\nu$-dimensional model precisely, consider a box $\Lambda$ with sides of length $L_{1}, \ldots, L_{\nu}$. At each point, $\alpha$, with integral coordinates in $\Lambda$ we have an independent quantum spin $\overrightarrow{\mathbf{s}}_{\alpha}$ (with three components $s_{\alpha}^{(i)}$ ) of some fixed angular momentum $S\left(=\frac{1}{2}, 1, \ldots\right)$. The basic Hamiltonian is given $b y^{6}$

$$
H_{\Lambda}=\sum_{\alpha \in \Lambda} \sum_{i=1}^{\nu}\left(S^{2}-\overrightarrow{\mathbf{S}}_{\alpha} \cdot \overrightarrow{\mathbf{S}}_{\alpha+\delta_{i}}\right),
$$

where $\delta_{i}$ is the unit vector with $i$ th component +1 and we intend periodic boundary conditions, i.e., if $\alpha$ is at a face so that $\alpha+\delta_{i} \in \Lambda, \alpha+\delta_{i}$ is interpreted as $\alpha-\left(L_{i}-1\right) \delta_{i}$. Each pair of points is counted once in (1). In the box $\Lambda$, one forms the Gibbs state

$$
\langle A\rangle_{\Lambda}=\operatorname{Tr}\left[A \exp \left(-\beta H_{\Lambda}\right)\right] / \operatorname{Tr} \exp \left(-\beta H_{\Lambda}\right) .
$$

We will prove directly that for $\beta>\tilde{\beta}_{\mathrm{c}}(S)$ (to be defined below),

$$
\lim _{\Lambda \rightarrow \infty}|\Lambda|^{-2}\left\langle\left[\sum_{\alpha \in \Lambda} \overrightarrow{\mathbf{s}}_{\alpha}\right]^{2}\right\rangle>0
$$

which is one notion of phase transition. It is not difficult $^{7}$ to go from (3) to other notions of phase transition such as nondifferentiability of the free energy or discontinuity of magnetization in external field. Our upper bound, $\tilde{\beta}_{c}(S)$, on the critical $\beta$ is given by the implicit equation

$$
\sqrt{\frac{2}{3}}(S+1)=(2 \pi)^{-\nu} \int \operatorname{coth}\left(\sqrt{\frac{2}{3}} \tilde{\beta}_{c} E_{p} S\right) d^{\nu} p,
$$

where

$$
E_{p}=\nu-\cos p_{1}-\ldots-\cos p_{\nu}
$$

and $2 S E_{p}$ is the energy of a momentum $p$ spin wave; the integral is over the hypersphere $\left|p_{i}\right|$ $\leqslant \pi, i=1, \ldots, \nu$. Since $\operatorname{coth} x$ is monotone decreasing and goes to 1 as $x \rightarrow \infty$, it is not hard to see that (4a) has a finite solution as long as $\nu \geqslant 3$ (in order that the integral converge at $p=0$ ) and the parameter $S$ obeys $S+1>\sqrt{\frac{3}{2}}$ (i.e., for all physical values $S=\frac{1}{2}, 1$, etc.). In Table I, we list for $\nu=3$ the function $\mu(S) \equiv \tilde{\beta}_{c}(S) S(S+1)$ obtained by a numerical analysis. ${ }^{8}$ It is easy to deduce from
TABLE I. The second column lists our rigorous upper bounds on inverse transition temperatures in three dimensions. The third column lists $\beta_{c}(S) S(S+1)$ according to Ref. 1 .

\begin{tabular}{ccc}
\hline \hline & $\mu(S) \equiv \tilde{\beta}_{c}(S) S(S+1)$ & $\begin{array}{c}\text { Series expansion value } \\
\text { (Ref. 1) }\end{array}$ \\
\hline $1 / 2$ & 1.354 & $0.8880 \pm 0.0009$ \\
1 & 0.965 & $0.7660 \pm 0.0020$ \\
$3 / 2$ & 0.872 & $0.7308 \pm 0.0013$ \\
2 & 0.832 & $0.7084 \pm 0.0006$ \\
$5 / 2$ & 0.810 & \\
3 & 0.797 & \\
$7 / 2$ & 0.788 & \\
4 & 0.783 & \\
$9 / 2$ & 0.778 & $0.6916 \pm 0.0002$ \\
5 & 0.775 & \\
$\infty$ & 0.758 & \\
\hline
\end{tabular}

the convexity of $\operatorname{coth} x$ that $\nu \tilde{\beta}_{c}(S)$ is a decreasing function of $\nu$, as it should be.

The variable $\mu$ is convenient since $\mu(S)$ has a finite limit $\mu(\infty)=\frac{3}{2}(2 \pi)^{-\nu} \int E_{p}^{-1} d p$. This is reasonable on the basis of limit theorems ${ }^{9}$ which relate the limit $S \rightarrow \infty$ to the classical Heisenberg model, i.e., a model with Hamiltonian (1) but with $\overrightarrow{\mathbf{S}}_{\alpha}$ a vector on a sphere of radius $S=1$ rather than an operator. It has recently been proven by Fröhlich, Simon, and Spencer ${ }^{10}$ (henceforth FSS) that this classical lattice system has a phase transition-their upper bound on the critical $\beta$ is just $\mu(\infty)$ which is also the transition temperature for the spherical model. The starting point of our proof is the basic strategy of FSS; namely in the classical case, one proves the following two things.

(A) An upper bound on the Fourier transform of $\left\langle\overrightarrow{\mathbf{s}}_{\alpha} \cdot \overrightarrow{\mathbf{S}}_{\beta}\right\rangle$ of the following form: Let

$$
\hat{s}_{p}^{(j)}=|\Lambda|^{-1 / 2} \sum_{\alpha \in \Lambda} s_{\alpha}^{(j)} e^{i p \cdot \alpha}
$$

for $p$ obeying $p_{i} L_{i}=2 \pi n_{i}\left(n_{i}=-\frac{1}{2} L_{i}+1, \ldots, \frac{1}{2} L_{i}\right.$ if $L_{i}$ is even). Then, in the classical case, FSS show that

$$
\sum_{j=1}^{3}\left\langle\hat{s}_{p}{ }^{(j)} \hat{s}_{-p}{ }^{(j)}\right\rangle \leqslant \frac{3}{2 \beta E_{p}} .
$$

(B) A trivial equality (Parseval),

$$
|\Lambda|^{-1} \sum_{p, j}\left\langle\hat{s}_{p}{ }^{(j)} \hat{s}_{-p}{ }^{(j)}\right\rangle=\left\langle\vec{S}_{0} \cdot \vec{S}_{0}\right\rangle=S^{2} \text {. }
$$

Equations (5) and (6) together imply that for $S^{2} \beta$ $>\mu(\infty)$,

$$
\lim _{|\Lambda| \rightarrow \infty}|\Lambda|^{-1}\left\langle\hat{s}_{0}^{2}\right\rangle>0
$$


[Note that $\left\langle\hat{s}_{0}{ }^{2}\right\rangle=|\Lambda|^{-1}\left\langle\left[\sum_{\alpha \in \Lambda} s_{\alpha}\right]^{2}\right\rangle$.]

In carrying this strategy to the quantum case, the first problem that arises is that (5) can no longer hold, for (5) would imply that with $\Lambda$ fixed and $\beta \rightarrow \infty,\left\langle\overrightarrow{\mathrm{s}}_{\alpha} \cdot \overrightarrow{\mathrm{s}}_{\gamma}\right\rangle$ would approach a constant. But it does not; it approaches $S^{2}$ if $\alpha \neq \gamma$ and $S(S$ +1 ) if $\alpha=\gamma$. The key to solving this problem is to deal with a different, but natural, two-point function. Define the Duhamel two-point function of $A$ and $B$ by

$$
\begin{aligned}
(A, B) & \equiv\left[\operatorname{Tr}\left(e^{-\beta H}\right)\right]^{-1}\left[\int_{0}^{1} d t \operatorname{Tr}\left(A e^{-t \beta H} B e^{-(1-t) B H}\right)\right] \\
& =\left.\frac{\partial^{2}}{\partial \mu \partial \lambda} \operatorname{Tr}\left(e^{-\beta H+\mu A+\lambda B}\right)\right|_{\mu=\lambda=0}\left[\operatorname{Tr}\left(e^{-\beta H}\right)\right]^{-1} .
\end{aligned}
$$

This natural object written in a different form is the inner product used in Bogoliubov's inequality. ${ }^{11}$ It has been discussed recently by several authors in this form. ${ }^{12,13}$

The analog to step (A) in our quantum proof ${ }^{7}$ is to show that, if $L_{1}, \ldots, L_{\nu}$ are even,

$$
\sum_{j=1}^{3}\left(\hat{s}_{p}{ }^{(j)}, \hat{s}_{-p}{ }^{(j)}\right) \leqslant \frac{3}{2 \beta E_{p}} .
$$

The proof of $\left(5^{\prime}\right)$ is related to the FSS proof of (5) with two important changes: (a) We must avoid use of the usual kind of transfer matrix which exists in the classical case but not the quantum case. (b) We must extend a certain integral inequality to the noncommutative case.
Having overcome the first difficulty and established $\left(5^{\prime}\right)$ we are faced with another; the analog of $(6)$ is

$$
|\Lambda|^{-1} \sum_{p, j}\left\langle\hat{s}_{p}^{(j)} \hat{s}_{-p}{ }^{(j)}\right\rangle=S(S+1) .
$$

$\left(5^{\prime}\right)$ involves the Duhamel two-point function while $\left(6^{\prime}\right)$ involves the ordinary thermal twopoint function. The usual inequality ${ }^{11}$

$$
(A *, A) \leqslant \frac{1}{2}[\langle A * A\rangle+\langle A A *\rangle],
$$

goes in the wrong direction. The second step in our proof ${ }^{7}$ is a generalization ${ }^{14}$ of an inequality of Roepstorff. ${ }^{13}$ Let $f(x)$ be the convex function for $x \geqslant 0$ given by

$$
f(x \tanh x)=x^{-1} \tanh x .
$$

Then

$$
\left(A^{*}, A\right) \geqslant \frac{1}{2}\left[\langle A * A\rangle+\left\langle A A^{*}\right\rangle\right] f(c),
$$

where

$$
c=\beta\langle[A *,[H, A]]\rangle / 2[\langle A * A\rangle+\langle A A *\rangle] .
$$

(8) should be applicable to more than just our problem. It is the necessary ingredient needed to obtain a bound on the thermal two-point function from $\left(5^{\prime}\right)$. The proof of (8) relies on the fact that $f$ is convex and that the integrand in the definition of the Duhamel two-point function $\left(A^{*}, A\right)$ is the Laplace transform of a positive measure.

Using (8) and (5'), properties of $f$, and the elementary bound

$$
\beta \sum_{j=1}^{3}\left\langle\left[\tilde{s}_{p}^{(j)},\left[H, \tilde{s}_{-p}{ }^{(j)}\right]\right]\right\rangle=4 \beta E_{p}(|\Lambda| \nu)^{-1} \sum_{\alpha \in \Lambda} \sum_{i=1}^{\nu}\left\langle\overrightarrow{\mathbf{s}}_{\alpha} \cdot \overrightarrow{\mathbf{s}}_{\alpha+\delta_{i}}\right\rangle \leqslant 4 \beta E_{p} S^{2},
$$

one has for $p \neq 0$ that

$$
\begin{aligned}
& \left\langle\hat{s}_{-p} \bullet \hat{s}_{p}\right\rangle \leqslant S \sqrt{\frac{3}{2}} \operatorname{coth} x_{p}, \\
& x_{p}=\sqrt{\frac{2}{3}} \beta S E_{p} .
\end{aligned}
$$

Equation ( $\left.5^{\prime \prime}\right)$ is the quantum analog of (5). $\left(6^{\prime}\right)$ and $\left(5^{\prime \prime}\right)$ imply

$$
|\Lambda|^{-1} \sum_{p \neq 0}\left\langle\hat{s}_{p} \cdot \hat{s}_{-p}\right\rangle \leqslant \sqrt{\frac{3}{2}} S|\Lambda|^{-1} \sum_{p \neq 0} \operatorname{coth} x_{p} .
$$

As $|\Lambda| \rightarrow \infty$, (4) is obtained.

We close with two related remarks. It seems to us that in the second part, (B), of our proof we have neither given away any factors nor have we had to make any restrictions on the model. However, in the first part, (A), our method of proof has restricted us to nearest-neighbor coupling and a simple cubic lattice. The factor of $\frac{3}{2}$ can be interpreted as coming from $\frac{1}{2} k T$ per component summed over three components. Since the components are related, there are really only two degrees of freedom, so $\frac{3}{2}$ might become $\frac{2}{2}$ $=1$ at low temperatures.

It is a pleasure to thank J. Fröhlich for valuable correspondence and J. Barnes for doing the numerical calculations appearing in Table $\mathrm{I}$.

\footnotetext{
*Research supported by U. S. National Science Foundation Grant No. GP-40768X.

$\dagger$ Research supported by U. S. National Science Foundation Grant No. MCS 75-21648.

$\$$ Alfred Sloan Fellow. Research supported by U. S. National Science Foundation Grant No. GP-39048.

${ }^{1}$ D. S. Ritchie and M. E. Fisher, Phys. Rev. B $\underline{5}, 2668$ (1972).

${ }^{2}$ See, e.g., F. Bloch, Z. Phys. 61, 206 (1930); or F. Dyson, Phys. Rev. 102, 1217, 1230 (1956).
} 
${ }^{3}$ T. Asano, Phys. Rev. Lett. 24, 1409 (1970).

${ }^{4}$ N. D. Mermin and H. Wagner, Phys. Rev. Lett. 17, 1133 (1966); P. C. Hohenberg, Phys. Rev. $\underline{158}, 383$ (1967).

${ }^{5}$ J. Ginibre, Commun. Math. Phys. 14, 205 (1969); D. Robinson, Commun. Math. Phys. 14, 195 (1969).

${ }^{6}$ We normalize $\boldsymbol{H}$ so that the ground-state energy is zero. A factor $J$ which is often introduced can be absorbed into $\beta$.

${ }^{7}$ This and other details will appear in a longer paper by the authors.

${ }^{8} \mathrm{~J} . \mathrm{F}$. Barnes, private communication. The quantity $(2 \pi)^{-3} \int E_{p}^{-1} d^{3} p=0.5054620197$ has been computed by G. N. Watson, Q. J. Math. 10, 266 (1939). One can integrate the nonsingular quantity coth $a E_{p}-\left(a E_{p}\right)^{-1}$ on a computer.

${ }^{9}$ K. Millard and H. Leff, J. Math. Phys. (N.Y.) 12,
1000 (1971); E. H. Lieb, Commun. Math. Phys. 31, 327 (1973).

${ }^{10} \mathrm{~J}$. Fröhlich, B. Simon, and T. Spencer, Phys. Rev. Lett. 36, 804 (1976); and "Infrared Bounds, Phase Transitions and Continuous Symmetry Breaking" (to be published)

${ }^{11}$ See, e.g., D. Ruelle, Statistical Mechanics (Benjamin, New York, 1969).

${ }^{12} R$. Powers, to be published.

${ }^{13} \mathrm{G}$. Roepstorff, Commun. Math. Phys. 46, 253 (1976).

${ }^{14}$ Equation (8) with $f(c)$ replaced by $c^{-1}\left(1-e^{-c}\right)$ and $A$ $=A^{*}$ appears in Ref. 13. Since one can show that $f(c)$ $\geqslant c^{-1}\left(1-e^{-c}\right),(8)$ improves the result of Ref. 13 in two ways. Where we use the fact that the integrand in the definition of $\left(A^{*}, A\right)$ is the Laplace transform of a positive measure, Roepstorff uses the weaker fact that the integrand is log convex.

\title{
Threshold Production of Positive Pions and the Form of the $\pi N N$ Vertex*
}

\author{
J. V. Noble \\ Department of Physics, University of Virginia, Charlottesville, Virginia 22091
}

(Received 3 March 1976)

Recent measurements of $\left(p, \pi^{+}\right)$reactions near threshold allow us to restrict substantially the possible forms of the empirical nonrelativistic $\pi N N$ vertex.

The theoretical description of $\left(p, \pi^{+}\right)$reactions is presently dominated by two disparate viewpoints: The first ${ }^{1}$ supposes that the incident proton shakes off a pion and the resulting neutron is captured into a single-particle orbital of the residual nucleus, a process which is analogous to $(d, p)$ stripping and is therefore called pionic stripping. The average influence of the target nucleons is taken into account through distortion of the incident and outgoing waves. The second $\mathrm{school}^{2}$ supposes that at the large momentum transfers of pionic stripping, single-nucleon wave functions have too little strength, so that there is a substantial gain to be made through sharing the momentum equally among several nucleons (incoherent multinucleon model). In a recent review I have discussed in detail my reasons for preferring the pionic stripping to the incoherent multinucleon model. ${ }^{3} \mathrm{As}-$ suming the former to be correct, there nevertheless remains a nontrivial problem of distinguishing the effect of coherent rescattering (distortion) from those of the nuclear structure as represented by the single-particle wave function. In trying to disentangle these effects it is essential to know the form of the $\pi N N$ vertex amplitude. Unfortunately, as several authors have shown, the nonrelativistic reduction of the $\pi N N$ coupling is made ambiguous by our ignorance of the "small" components of (four-component) nucleon wave functions in the usual nuclear-structure theories. ${ }^{4}$

The object of this note is to explore to what extent the phenomenological form of the $\pi N N$ vertex is fixed by our present experimental knowledge. The measurements at $185 \mathrm{MeV}^{5}$ of ${ }^{40} \mathrm{Ca}\left(p, \pi^{+}\right){ }^{41} \mathrm{Ca}$ (g.s.) and ${ }^{10} \mathrm{~B}\left(p, \pi^{+}\right){ }^{11} \mathrm{~B}$ (g.s.), when combined with more recent results at $154-\mathrm{MeV}$ bombarding energy, ${ }^{6}$ tend to distinguish relatively clearly between the two most popular phenomenological nonrelativistic $\pi N N$ effective Hamiltonians. To see how this comes about, we note first that the amplitude for pionic stripping, in the distorted-wave Born approximation, has the form

$$
\int d^{3} x \varphi_{\overrightarrow{\mathrm{k}}^{\prime}}^{(-) *(\overrightarrow{\mathrm{x}})}\left\langle f\left|\bar{\psi}_{n}(\overrightarrow{\mathrm{x}}) O \psi_{p}(\overrightarrow{\mathrm{x}})\right| \bar{\Psi}_{i, \overrightarrow{\mathrm{p}}^{(+)}}\right\rangle,
$$

where

$$
\begin{array}{r}
\left.\left|\bar{\Psi}_{\left.i, \overrightarrow{\mathrm{p}}^{(+)}\right\rangle=\left[1+(E+i \eta-H)^{-1}\left(H-V_{\text {coul }}-E\right)\right]} \times a^{\dagger}(\overrightarrow{\mathrm{p}})\right| i\right\rangle
\end{array}
$$

is the solution of the Schrödinger equation with asymptotic Coulomb-wave behavior; $a^{\dagger}(\overrightarrow{\mathrm{p}})$ is a creation operator for the incident proton, with barycentric momentum $\overrightarrow{\mathrm{p}} ; \varphi_{\mathrm{k}^{\prime}}{ }^{(-)} *(\overrightarrow{\mathrm{x}})$ is the scattering wave function of the outgoing pion, which has 